\title{
Study and Management of a $Q$ Fever Outbreak among Machine Tool Workers in the Basque Country (Spain)
}

\author{
Jesús Delgado Naranjo, ${ }^{1,2}$ Eva Alonso Fustel, ${ }^{3}$ Inmaculada Aspiritxaga Gamarra, ${ }^{3}$ \\ Guillermo Ezpeleta Lobato, ${ }^{4}$ and Nerea Muniozguren Agirre $^{3}$ \\ ${ }^{1}$ Preventive Medicine Unit of Cruces Hospital, Basque Health Service OSAKIDETZA, 48903 Barakaldo, Bizkaia, Spain \\ ${ }^{2}$ Department of Preventive Medicine and Public Health, Faculty of Medicine, University of the Basque Country, 48940 Leioa, \\ Bizkaia, Spain \\ ${ }^{3}$ Unit of Epidemiology of Bizkaia, Sub-Directorate of Public Health of Bizkaia, Health Department of The Basque Government, \\ 48008 Bilbao, Bizkaia, Spain \\ ${ }^{4}$ Laboratory of Microbiology, Basurto Hospital, Basque Health Service OSAKIDETZA, 48013 Bilbao, Bizkaia, Spain
}

Correspondence should be addressed to Jesús Delgado Naranjo, iosudelgado@orange.es

Received 9 November 2010; Revised 30 December 2010; Accepted 22 January 2011

Academic Editor: Leo J. Schouten

Copyright (C) 2011 Jesús Delgado Naranjo et al. This is an open access article distributed under the Creative Commons Attribution License, which permits unrestricted use, distribution, and reproduction in any medium, provided the original work is properly cited.

\begin{abstract}
The aim of this study is to describe a Q fever outbreak that affected the staff of a machine-tool factory in the Basque Country between 2009/12/20 and 2010/02/23. Study subjects were interviewed using a Q fever specific questionnaire and tested for Q fever serology (immunofluorescence assay with phase II antigen) and detecting Coxiella burnetii DNA using real-time PCR. We interviewed and tested 40 employees (90\% of the staff). 33 employees, all of them men, had positive serology (attack rate $82.5 \%$, 95\% CI: 70.2-94.8). Mean age was 43.7 years (95\% CI: $38.7-48.7$ ) in positive men, 33.7 years (95\% CI: $-16.6-83.9$ ) in negative men, and 36.25 (95\% CI: 27.5-45.0) in women (all negatives). 15 cases (45.5\%) were asymptomatic, 9 (27.3\%) had flu-like symptoms, and the other $9(27.3 \%)$ had developed radiologically confirmed pneumonia. We obtained 28 blood samples, 22 faeces samples, 11 milk samples, and one vaginal swab from 28 goats resting in a stable near the factory. Serology was positive in 18 goats (64.3\%). All environmental samples were negative.
\end{abstract}

\section{Introduction and Background}

Q fever is a ubiquitous zoonosis caused by Coxiella burnetii, an obligate intracellular coccobacillus, whose incubation period varies from two to six weeks, depending on the infective dose and host. Most cases are asymptomatic $(60 \%)$ or have mild flu-like symptoms. Only $20 \%$ of infected patients seek medical attention, 2 to $3 \%$ are admitted to a hospital and the case fatality ratio is 1 to $2 \%[1,2]$. Symptomatic patients present with a sudden onset of high fever, chills, severe headache, and dyspnoea. In some patients, the clinical course is complicated by pericarditis, myocarditis, pancreatitis, or haemolytic anaemia. A small proportion of infected patients develop chronic Q fever. Endocarditis is the most frequent complication, about $1 \%$ of patients, following $\mathrm{Q}$ fever. The most important reservoir are sheep, goats, and cattle.
Domestic animals such as dogs, cats, rabbits, and birds can also carry the disease. Ticks are thought to spread the disease among animals. Infected animals excrete the bacteria in milk, faeces, urine, and in high concentrations in amniotic fluid and the placenta. The animals are often asymptomatic except for increased incidence of spontaneous abortion. Infection in humans occurs through inhalation of contaminated aerosols or ingestion of unpasteurised milk. C. burnetii is resistant to chemical and physical disinfectants. A small inoculums is sufficient to cause clinical illness. Human-to-human transmission does not usually occur, although it has been described following contact with infected parturient women.

In The Basque Country, geographically located in the North of Spain, human Q fever is a notifiable disease, considered to be an endemic area of Q fever [3-5]. Several outbreaks have been recently reported in The Netherlands, 
Scotland, Slovenia, and other European countries [6-9]. In Bizkaia, there is a surveillance system in which all declared $\mathrm{Q}$ fever cases are systematically followed and studied. In the last four years, the $\mathrm{Q}$ fever incidence rate fluctuated from 8.44 cases per 100,000 in 2006 to 3.40 cases per 100,000 in 2009 [10]. Except for two outbreaks in 2008 (involving 7 cases) and one outbreak in 2007 (involving 4 cases), all declared cases were sporadic. Since Bizkaia is considered as an endemic area for $\mathrm{Q}$ fever, both medical practitioners and The Epidemiologic Surveillance Unit of Bizkaia are specially aware of $\mathrm{Q}$ fever. A recent study about Coxiella burnetii seroprevalence among animals in the Basque Country [11] has showed that the prevalence in sheep was $11.8 \%, 5.9 \%$ in goats, and $6.7 \%$ in beef cattle. Moreover, no special animal control measures are being taking by Veterinary and Public Health authorities.

In this paper, we report the results of the investigation of an outbreak among workers of a machine-tool factory, located in Bizkaia, a province of The Basque Country.

\section{Methods}

2.1. Outbreak Study Design. On 11 February 2010, primary care services notified to the Epidemiologic Surveillance Unit of Bizkaia two cases of atypical pneumonia. Since both of them worked at the same factory, we contacted with the company manager, who informed that 7 workers more had taken sick leave due to respiratory pathology. Moreover, it was known that the factory was located in a rural area and there was a goat stable around. The nine cases were tested to $\mathrm{Q}$ fever resulting all of them positive. The first case was detected on 2009/12/20 and the last one on 2010/02/23. Consequently, on $2010 / 03 / 02$, to evaluate the extent of the spread of the infection, a seroepidemiological survey of persons working in the factory was conducted. Besides, nearby goats were serologically tested, using the laboratory methods described below.

2.2. Study Subjects and Laboratory Analysis. Study subjects, who voluntary gave their informed consent, were interviewed using a $\mathrm{Q}$ fever specific questionnaire that included questions on animal exposure, food history (including consumption of unpasteurised milk), risk activities, work details, tick exposure, and places visited. A 5-mL blood sample was obtained from study subjects in order to test Q fever serology status and C. burnetii DNA presence. Serology was performed using an immunofluorescence assay (IFA) with phase II antigen, determining both IgM and IgG antibodies, using commercially available antigens (bioMérieux, France). Molecular detection of C. burnetii DNA was performed using real-time PCR. Target sequence for real-time PCR was the multicopy insertion element IS11-11 and Com1 gene, known to be species-specific for Coxiella burnetii [12].

A case was defined as a person who had positive serology for C. burnetii and/or had a positive PCR test. A titer of 200 or greater IgG and 80 or greater for IgM against phase II antigen were considered as significant positive serology [12].
Moreover, collection of animal samples (blood and milk samples and vaginal swabs) was obtained for PCR and serology analysis. Sera of animals were tested for the presence of anti-C. burnetii antibodies by means of an ELISA test (ELISA Cox kit, LSI-Laboratoire Service International, Lyon, France) according to manufacturer's instructions. Environmental presence of $C$. burnetii was tested by means of PCR from manure and aerosol samples.

2.3. Statistical Analysis. Proportions were compared using Pearson's Chi ${ }^{2}$ test or Fisher's exact test while continuous data were compared by means of Student $t$-test, using STATA 10.1 and SPSS 17.0. A difference was considered statistically significant when $P$ was less than .05 . All information was collected and processed according to Spanish personal data protection law and Spanish biomedical research law.

\section{Results}

The factory was isolated and set up in a rural and agricultural area, surrounded by numerous pastures where goats grazed. The plant was divided into two sections: (i) an administrative area with offices which faces a road and (ii) a mechanic workshop, faced to an open area near a stable with goats (Figure 1). There was continuous airflow from the stable towards the mechanic workshop. Just besides the workshop and separated by a wall was the stable with the animals.

At the moment of the study, the factory staff was made up of 44 persons, $91 \%$ of which were men. We interviewed and tested 40 employees ( $90 \%$ of the staff), 4 employees rejected to take part into the study. Among those who participated in the study, $36(90 \%)$ were men. The average age of men was 42.8 years ( $95 \%$ CI: $37.9-47.8$ ) and 36.3 years (95\% CI: 27.5-45.0) among women. 33 employees (all men) worked at the workshop area, while 7 employees ( 4 women and 3 men) worked at administrative section. Among those who worked at administrative section, the three men spent part of their working time at the workshop section. Consequently, due to the factory structure, which was divided into two sections, three levels of risk were found among workers: (i) highrisk employees, who worked all the time at workshop area which was near the stable with the goats, (ii) medium-risk employees, who worked at administrative area but used to go sporadically to the workshop, and (iii) low-risk employees who were all the time at administrative area, completely isolated from infected area (Table 1).

Overall, 33 employees had positive serology, representing, according to case definition, an attack rate of $82.5 \%$ (95\% CI: 70.2-94.8). At the moment of the study, all subjects were negative to PCR analysis. On the other hand, two subjects, at the beginning of the outbreak, had been tested in the hospital and their result had been PCR positive, coinciding with their acute symptomatic process. All of positive subjects were men. Mean age was 43.7 years (95\% CI: 38.7-48.7) in positive men, 33.7 years (95\% CI: - 16.6-83.9) in negative men, and 36.25 (95\% CI: 27.5-45.0) in women, all of them negatives. 


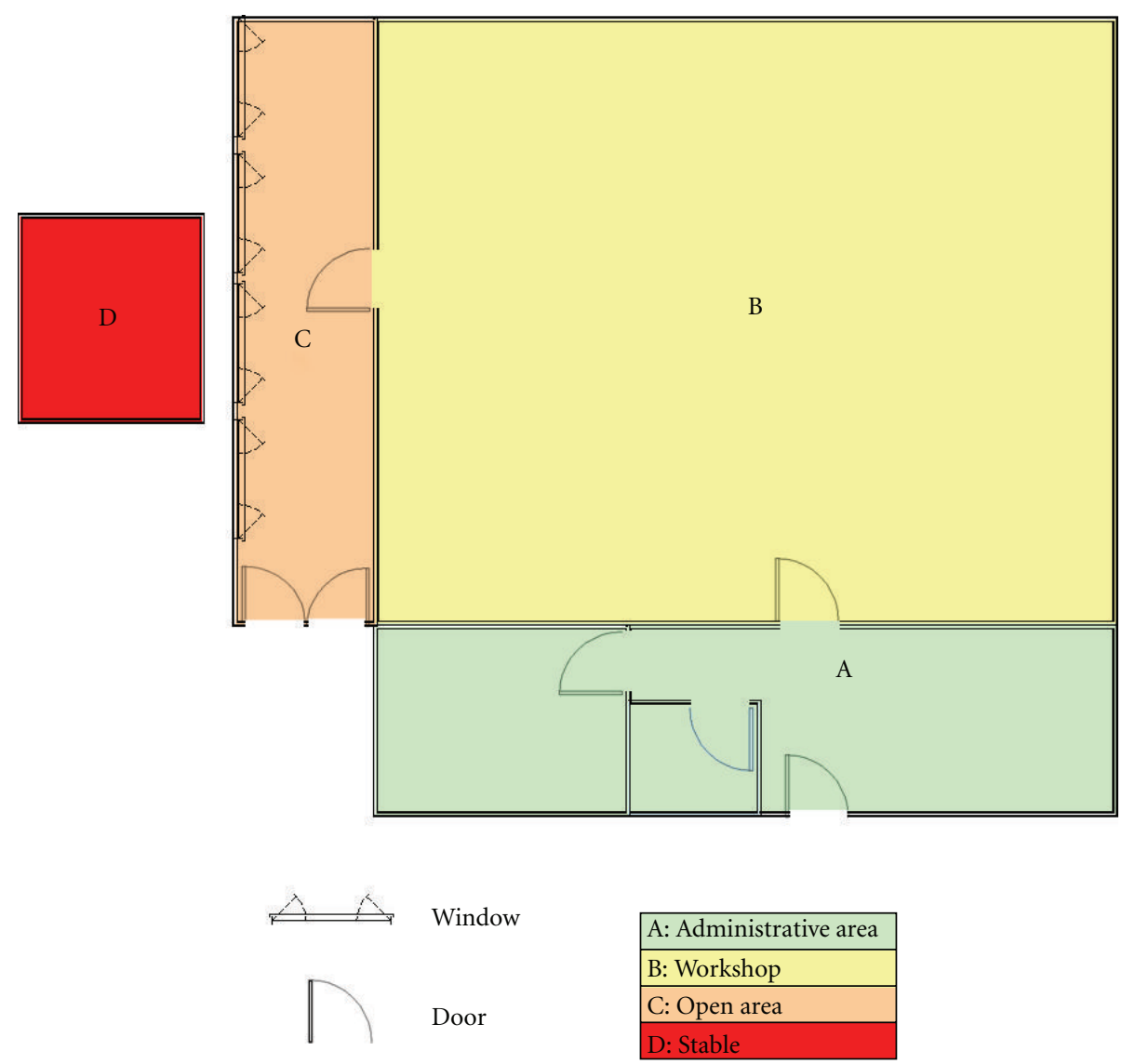

FIGURE 1: Plant plain of the factory showing its subdivision and the proximity to the stable.

TABle 1: Clinical manifestations, exposure levels, and socio-demographic findings of subjects.

\begin{tabular}{|c|c|c|c|c|c|c|c|c|c|c|c|c|c|}
\hline \multirow{3}{*}{ Clinical status } & & & & \multicolumn{4}{|c|}{ Sex } & \multicolumn{6}{|c|}{ Exposure level (2) } \\
\hline & \multicolumn{3}{|c|}{ Age } & \multicolumn{2}{|c|}{ Male } & \multicolumn{2}{|c|}{ Female } & \multicolumn{2}{|c|}{ High } & \multicolumn{2}{|c|}{ Medium } & \multicolumn{2}{|c|}{ Low } \\
\hline & $N$ & Mean & $95 \% \mathrm{CI}$ & $N$ & $\%$ & $N$ & $\%$ & $N$ & $\%$ & $N$ & $\%$ & $N$ & $\%$ \\
\hline \multicolumn{14}{|l|}{ Case (1) } \\
\hline Pneumonia & 9 & 43.3 & $33.3-53.4$ & 9 & 100 & - & - & 9 & 100 & - & 一 & - & - \\
\hline Flu-like symptoms & 9 & 39.4 & $27.9-50.9$ & 9 & 100 & - & - & 9 & 100 & - & 一 & 一 & - \\
\hline Asymptomatic & 15 & 46.4 & $38.4-54.4$ & 15 & 100 & - & - & 13 & 87 & 2 & 13 & - & - \\
\hline Noncase & 7 & 35.1 & $23.7-46.6$ & 3 & 43 & 4 & 100 & 2 & 29 & 1 & 14 & 4 & 100 \\
\hline Total & 40 & & & 36 & & 4 & & 33 & & 3 & & 4 & \\
\hline
\end{tabular}

(1) A case is considered when $\operatorname{IgG} \geq 200$ and $\operatorname{IgM} \geq 80$.

(2) Exposure level estimated according to workplace, distance to the workshop, and time spent at the workshop.

$N$ : number of subjects; 95\% CI: 95\% Confidence Interval.

From a total of 33 laboratory-confirmed cases, 15 (45.5\%) were asymptomatic, $9(27.3 \%)$ had flu-like symptoms, and other $9(27.3 \%)$ had developed radiologically confirmed pneumonia (Figure 2). The 7 employees whose serology was negative had no clinic symptoms. $100 \%$ of symptomatic subjects tested positive to Q fever. Among the 18 symptomatic cases, $13(72 \%)$ consulted their primary care physician and 7 (39\%) were attended by emergency services of hospitals but none of them required hospital admission. All cases developed clinical symptomatology between 20
December 2009 and 23 February 2010. All clinical cases showed Q fever common clinical presentation, and no fatal outcomes were detected.

We obtained 28 blood samples, 22 faeces samples, 11 milk samples and one vaginal swab from 28 goats grazing in the surroundings and resting in the stable near the factory. Serology was positive in 18 goats (64\%), while PCR analysis was negative in all animals. We also took two manure samples from the surroundings of the factory and two aerosol (air) samples inside the factory that were also negative. Since there 


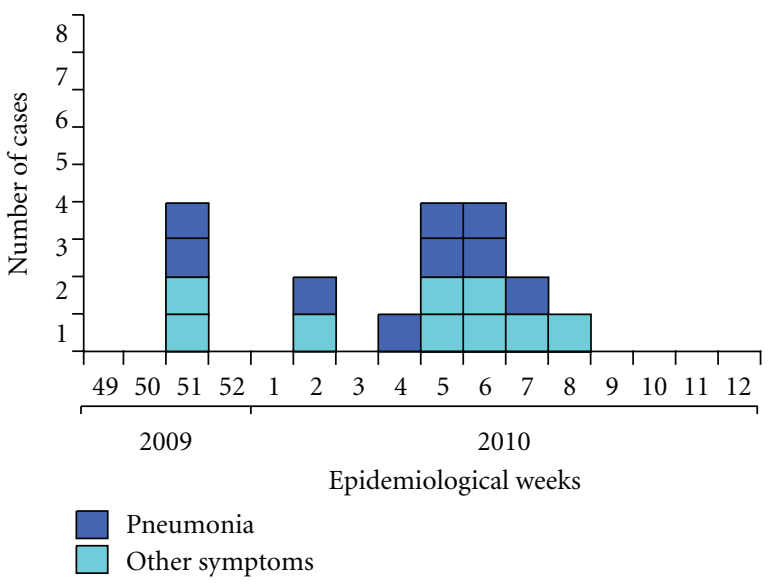

FIGURE 2: Epidemic curve of symptomatic infection cases involved in the Q fever outbreak.

was no other ruminant nearby, only these 28 goats were tested. All human $\mathrm{Q}$ fever cases coincided with the kidding or lambing season in the area.

Several control measures were taken: (i) all employees were given information about transmission, spread, and prevention of $\mathrm{Q}$ fever, (ii) both serologic and clinical control of affected subjects was performed, (iii) veterinary control, including test of goats as well as environmental samples, (iv) all the animals involved in the outbreak were sacrificed and the farming installation setup was removed following indications of veterinary services from Public Health Administration.

\section{Discussion}

This is a Q fever outbreak, in which its specific source is the existence of goats near the factory, where employees worked. Although there was not straight contact with the source, airborne transmission of C. burnetii appears to be the main way of transmission. In fact, this is the most frequent way of transmission to humans involving several outbreaks that have recently occurred in Europe [13, 14]. Furthermore, in this outbreak, cases are within the airborne spread area of $C$ burnetii and it coincides with the lambing season.

All subjects who tested positive to C. burnetii showed recent infection because their IgG and IgM titres are quite higher than cutoff for case definition. Indeed, IgM levels maintain high for 8 weeks after infection while IgG levels reach their maximum peak between 4 and 8 weeks after infection. The laboratory diagnosis criteria used in this survey (titers of 200 or greater IgG and 80 or greater for IgM against phase II antigen) satisfies the requirements to be considered recent cases of acute $\mathrm{Q}$ fever, providing a positive predictive value of $100 \%$ [12].

When we obtained the samples all subjects were asymptomatic, so the expected result was PCR negative, except for chronic Q fever cases. In spite of detecting chronic cases,
PCR was used to discard Q fever asymptomatic cases at the moment of the study. The obtained results are consistent with recent $\mathrm{Q}$ fever cases and nonchronic cases. From a prognostic point of view, at the moment of the study all subjects were PCR negative, suggesting that all of them had been recent cases of acute forms of $\mathrm{Q}$ fever rather than evolutive chronic Q fever [15].

All infected employees worked in the workshop section near the stable, and, therefore, they were more exposed to airborne spread. Those who worked at administrative area were far from the stable, and the probability of airborne spread was quite lower. These results are consistent with risk levels defined according to factory structure and work time of workers. In fact, high-risk employees who worked all the time at workshop area, which was near the stable with the goats, had the highest number of cases. A lower number of cases were detected among medium-risk employees, who worked at administrative area but used to go sporadically to the workshop. Finally, no case was detected among lowrisk employees who were all the time at administrative area, completely isolated from infected area. According to these risk areas, a risk gradient has been observed: the highest number of cases occurred among employees who worked full time in the workshop (31 cases), a shorter number of cases among employees who sporadically went to the workshop (2 cases), and no case was registered in the administrative area, which was completely isolated from infection source. Besides, 100\% of medium-risk cases were asymptomatic, while among high-risk employees, 55\% had showed clinical manifestations of Q fever and 27\% developed pneumonia.

Nevertheless there are studies that show a higher Q fever incidence in males $[13,14]$, the sex does not appear to be a relevant risk factor in this outbreak. In fact, the reason to explain that all females were noninfected is that their work placement was the administrative area, which was far from the infection source. Although age could be considered as a risk factor, in this study, the role of age is limited. There are not significant statistical differences between infected and noninfected subjects, in all clinic categories: pneumonia cases $(t=-1.27 ; P=.22)$, flu-like cases $(t=-0.61 ; P=.54)$, and asymptomatic cases $(t=-1.77 ; P=.09)$.

Other kind of exposure sources were dismissed because, apart from their exposure at workplace, none of the affected subjects showed other kind of $\mathrm{Q}$ fever risk factor as consumption of unpasteurised milk, risk activities, or tick exposure. Since there was no other ruminant nearby and the specific $\mathrm{Q}$ fever questionnaire was negative for other risk factors, only the tested goats can be considered as the most probable source of Q fever cases. Moreover, the factory is located in a rural mountainous and isolated area, so the shedding only affected to the workers of this factory.

The first $\mathrm{Q}$ fever outbreak among humans in The Basque Country was described in 1981 [5]. Although The Basque Country is considered to be an endemic area, this is the first time that the staff of a company, whose activity can be considered as at $\mathrm{Q}$ fever nonrisk, is affected by this infection. 


\section{Conclusions}

In conclusion, this outbreak highlights the fact that $\mathrm{Q}$ fever is capable of causing localized outbreaks in apparent nonexposed population, with high attack rates, even in nonhighrisk professionals, due to airborne rather than direct contact spread. The rapid identification of the cause of the outbreak due to high awareness of $\mathrm{Q}$ fever and good communication lines between clinicians and public health officials allowed rapid control measures of the outbreak, including drastic veterinary measures. Preventive measures against $Q$ fever are especially relevant in rural areas. Finally, this outbreak investigation emphasizes the importance of maintaining both a robust microbiology capability and collaboration between field epidemiology units, reference laboratories, and health primary care services.

\section{Conflict of Interests}

The authors declare no conflict of interests.

\section{Acknowledgments}

The authors would like to thank Microbiology Laboratory of Basurto Hospital, Work Health services of the factory, Work Health Institute (OSALAN), Unit of Preventive Medicine of Cruces Hospital, and the factory management and the factory staff for their collaboration and facilities provided.

\section{References}

[1] C. E. Delsing and B. J. Kullberg, "Q fever in the Netherlands: a concise overview and implications of the largest ongoing outbreak," Netherlands Journal of Medicine, vol. 66, no. 9, pp. 365-367, 2008.

[2] H. Tissot-Dupont and D. Raoult, "Q fever," Infectious Disease Clinics of North America, vol. 22, no. 3, pp. 505-514, 2008.

[3] J. M. Sanzo, M. A. Garcia-Calabuig, A. Audicanat, and V. Dehesa, "Q fever: prevalence of antibodies to Coxiella burnetii in the Basque Country," International Journal of Epidemiology, vol. 22, no. 6, pp. 1183-1188, 1993.

[4] M. Montejo Baranda, J. Corral Carranceja, and C. Aguirre Errasti, "Q fever in the Basque Country: 1981-1984," Reviews of Infectious Diseases, vol. 7, no. 5, pp. 700-701, 1985.

[5] E. C. Aguirre, B. M. Montejo, J. L. Hernandez Almaraz et al., "An outbreak of Q fever in the Basque country," Canadian Medical Association Journal, vol. 131, pp. 48-49, 1984.

[6] L. E. Wilson, S. Couper, H. Prempeh et al., "Investigation of a Q Fever Outbreak in a Scottish Co-Located Slaughterhouse and Cutting Plant, Zoonoses," Public Health, pp. 493-498, 2009.

[7] I. Karagiannis, G. Morroy, A. Rietveld et al., "Q fever outbreak in the Netherlands: a preliminary report," Euro Surveillance, vol. 12, no. 8, Article ID E070809.2, 2007.

[8] A. Medić, B. Dželalija, V. P. Polićo, I. G. Margan, B. Turković, and V. Gilić, "Q fever epidemic among employees in a factory in the suburb of Zadar, Croatia," Croatian Medical Journal, vol. 46, no. 2, pp. 315-319, 2005.

[9] "Q fever outbreak in Switzerland," $M M W R$, vol. 33, no. 25, pp. 355-361, 1984.
[10] “Dirección Territorial de Sanidad y Consumo de Bizkaia," Surveillance Review 2009, http://www.euskadi.net/contenidos/ informacion/publicaciones_epidem/es_4383/adjuntos/Bizkaia 2009_cas.pdf. 2010.

[11] F. Ruiz-Fons, I. Astobiza, J. F. Barandika et al., "Seroepidemiological study of Q fever in domestic ruminants in semiextensive grazing systems," BMC veterinary research, vol. 6, p. 3, 2010.

[12] M. Maurin and D. Raoult, "Q fever," Clinical Microbiology Reviews, vol. 12, no. 4, pp. 518-553, 1999.

[13] I. D. Aitken, K. Bogel, E. Cracea et al., "Q fever in Europe: current aspects of aetiology, epidemiology, human infection, diagnosis and therapy," Infection, vol. 15, no. 5, pp. 323-327, 1987.

[14] J. D. Hartzell, R. N. Wood-Morris, L. J. Martinez, and R. F. Trotta, "Q fever: epidemiology, diagnosis, and treatment," Mayo Clinic Proceedings, vol. 83, no. 5, pp. 574-579, 2008.

[15] O. A. Sukocheva, B. P. Marmion, P. A. Storm, M. Lockhart, M. Turra, and S. Graves, "Long-term persistence after acute $\mathrm{Q}$ fever of non-infective Coxiella burnetii cell components, including antigens," QJM, vol. 103, no. 11, pp. 847-863, 2010. 


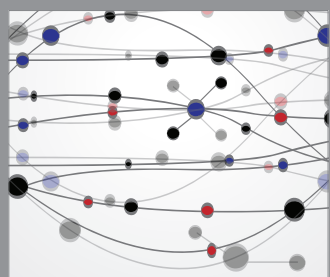

The Scientific World Journal
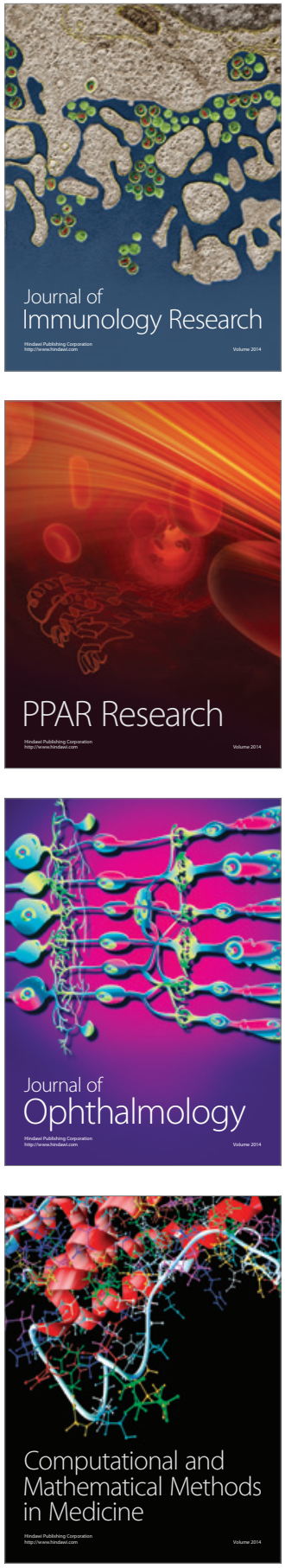

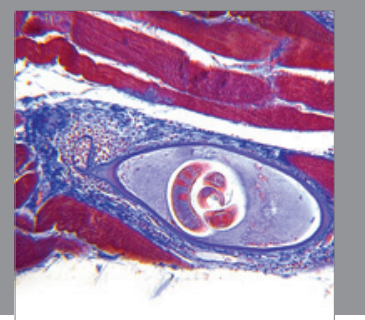

Gastroenterology

Research and Practice
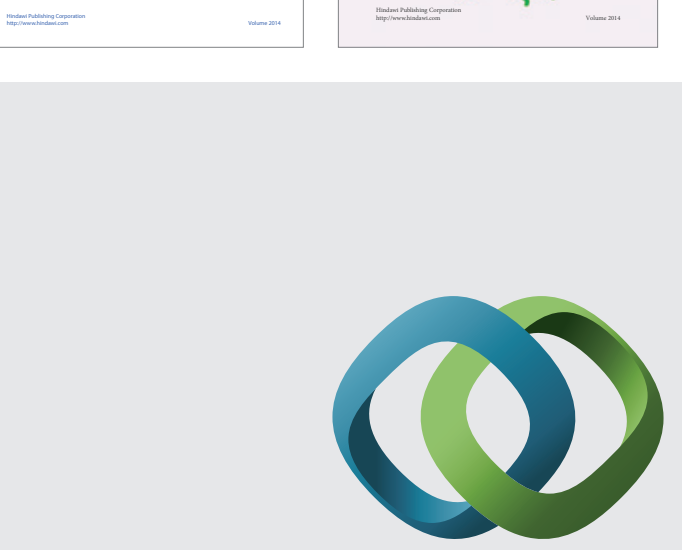

\section{Hindawi}

Submit your manuscripts at

http://www.hindawi.com
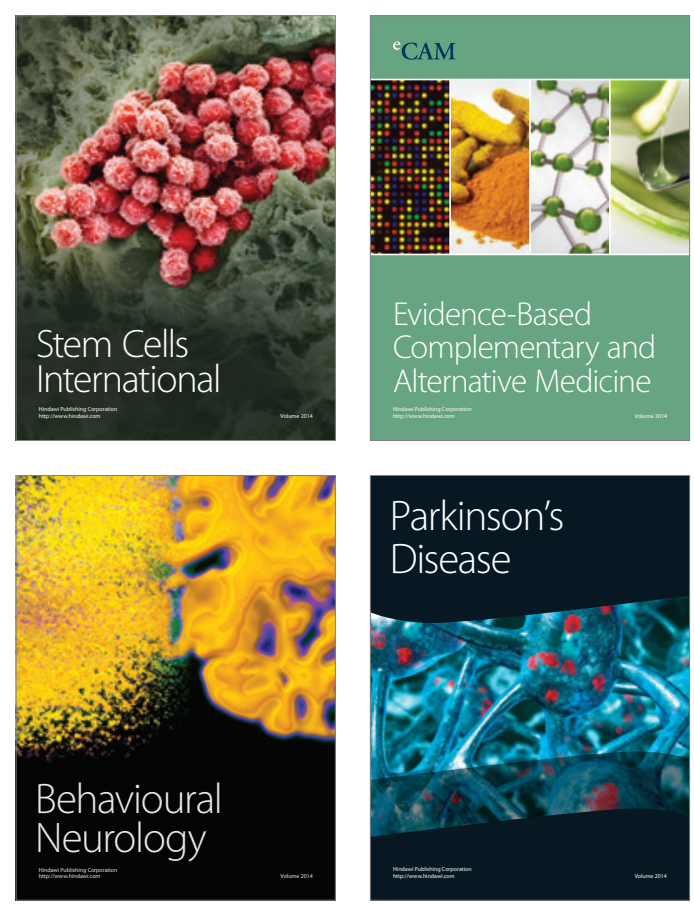

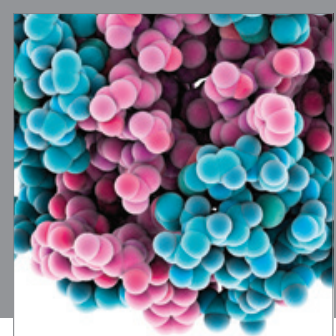

Journal of
Diabetes Research

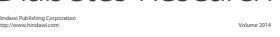

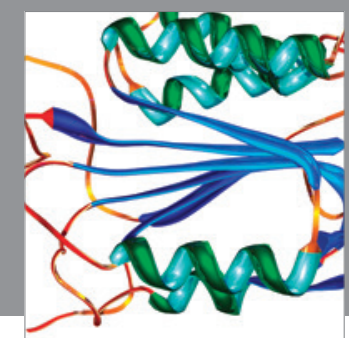

Disease Markers
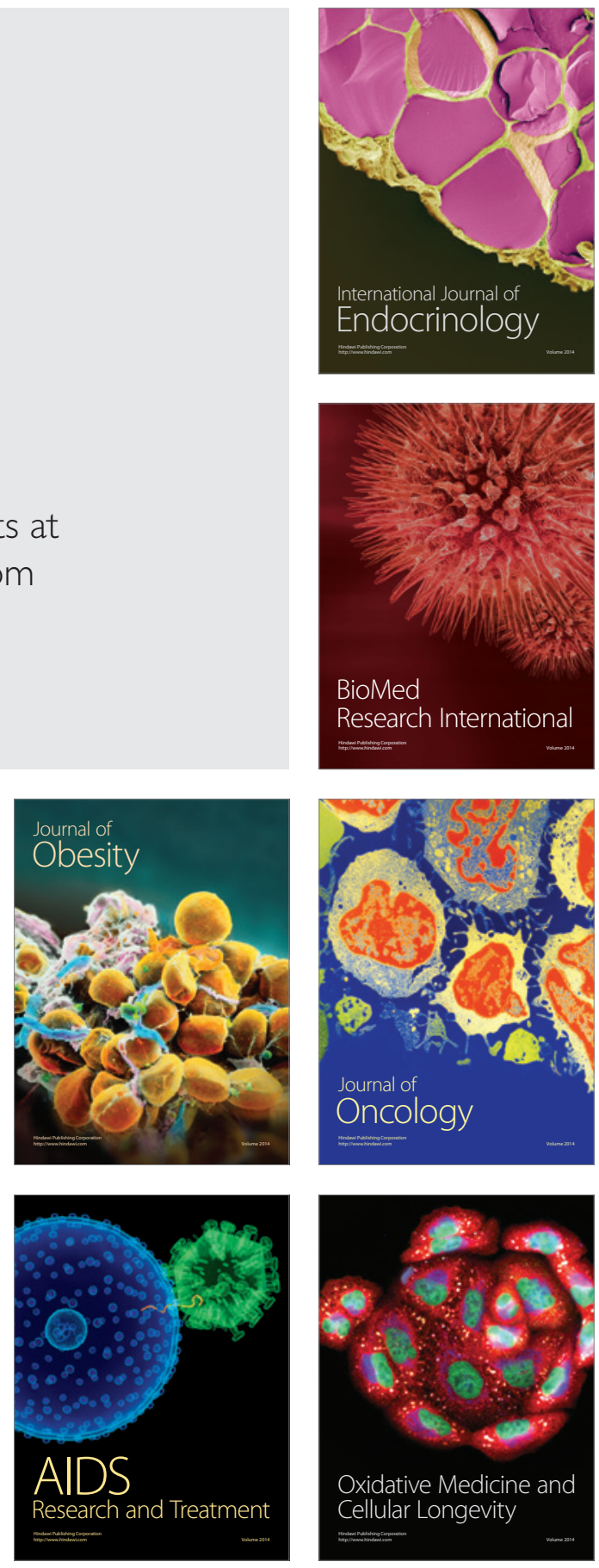